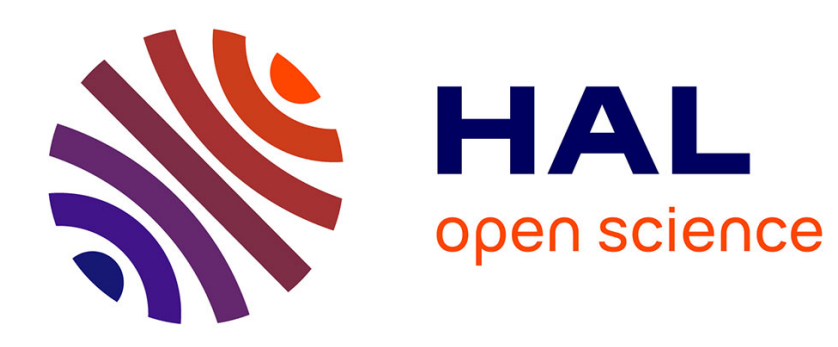

\title{
A Photothermoelectric Effect in Graphene
}

Denis Basko

\section{To cite this version:}

Denis Basko. A Photothermoelectric Effect in Graphene. Science, 2011, 334, pp.610. 10.1126/science.1214560 . hal-00642464

\section{HAL Id: hal-00642464 \\ https://hal.science/hal-00642464}

Submitted on 18 Nov 2011

HAL is a multi-disciplinary open access archive for the deposit and dissemination of scientific research documents, whether they are published or not. The documents may come from teaching and research institutions in France or abroad, or from public or private research centers.
L'archive ouverte pluridisciplinaire HAL, est destinée au dépôt et à la diffusion de documents scientifiques de niveau recherche, publiés ou non, émanant des établissements d'enseignement et de recherche français ou étrangers, des laboratoires publics ou privés. 


\title{
A photothermoelectric effect in graphene
}

\author{
D. M. Basko \\ Université Grenoble 1/CNRS, LPMMC UMR 5493, B.P. 166, 38042 Grenoble, France
}

One usually learns about thermocouples in the highschool physics course. Take pieces of two different metals, put them in contact, heat up the contact with a candle while keeping the opposite ends cold, and you will measure a voltage between the two cold ends. And if one takes two pieces of the same metal, will the voltage appear? Try to answer "yes" at a high school test, and you will shamefully fail. Nevertheless, the authors of the paper published on page 648 of this issue [1], managed to heat up a junction made of the same material, and measure a voltage. The material they used was graphene. So, does it mean that graphene violates the laws of physics that we have read about in high-school textbooks? Let us see in more detail.

How does a thermocouple work? When electrons move through a metal, they carry electric charge and energy. The former is responsible for the electric current, and the latter for the heat current. While the electric current is determined just by the total number of electrons transferred from one electrode (source) to the other one (drain), the heat current also depends on how the electrons are distributed among energy levels. Still, since both have the same physical origin, namely, motion of electrons through the metal, they are strongly connected. One manifestation of this is the Wiedemann-Franz law, relating electric and thermal conductivity. Another manifestation is the thermoelectric effect: apply a temperature difference to a sample, and you will measure a voltage. Indeed, temperature difference produces a heat current, accompanied by electric current (carried by the same electrons), which, by Ohm's law, is proportional to the voltage. The ratio between the voltage and the temperature difference is called thermopower or Seebeck coefficient, which depends on the material. If a junction of two metals is heated up, a voltage is generated in each metal. The net voltage across the sample is different from zero if the two samples have different Seebeck coefficients, and is zero if they are the same.

The key idea of the experiment is to take a single sheet of graphene, but to place two regions of it under different conditions, so that they have different Seebeck coefficients. It is possible because the density of electrons in graphene can be strongly modified by placing external gate electrode nearby, and applying a constant voltage to it. There is no electric contact between the gate and the graphene sheet, but the electric field from the gate penetrates the graphene, and can push electrons away from it, or, oppositely, attract more electrons, depending on the sign of the gate voltage. This does not work with ordinary metals, because the external electric field is screened at short distances of the order of atomic length, while charge and heat transport occurs in the bulk of the metal, and thus remain unaffected by the external field. But graphene itself is atomically thin, and this permits the giant field effect. This was the primary subject of the pioneering work on graphene, published 7 years ago [2]: one could change the electrical conductivity by orders of magnitude simply tuning the gate voltage. By virtue of the general Mott relation [3], a change in conductivity implies a change in the Seebeck coefficient, as was confirmed experimentally for the specific case of graphene [4]. At room temperature, the observed variation of the Seebeck coefficient amounted to a few tens of $\mu \mathrm{eV} / \mathrm{K}$, which is at least an order of magnitude higher than for a junction between two conventional metals (gold, silver, copper, etc.), and of the same order as for alloys used in thermocouples (chromel, alumel, constantan). Besides this large value, the crucial advantage of graphene is that it can be controlled by the external gate voltage.

So, using a combination of two gate electrodes with different voltages $V_{1}, V_{2}$, one produces two regions of graphene sheet with different electronic densities. The junction is heated up by a focused a laser beam. Thus, the voltage appears upon exposure of the sample to light. Actually, this perfectly matches the definition of the photovoltaic effect. Does it mean that the studied device is a photodiode rather than a thermocouple? In a conventional photodiode, one illuminates a semiconductor $p-n$ junction by light, which excites electrons and holes. The electric field, always present at the $p$ - $n$ junction, pulls the electrons and the holes on the opposite sides of the junction. The spatial separation of negative and positive charges results in a photovoltage. So, which mechanism is responsible for the photovoltage measured in Ref. 1? A similar question has been addressed for a junction between single-layer graphene and bilayer graphene [5], where the two mechanisms would result in the opposite sign of the photovoltage, and the one corresponding to the thermoelectric mechanism was observed. In the doubly-gated monolayer graphene of Ref. 1, the fieldinduced carrier separation would result in two different regions in the $\left(V_{1}, V_{2}\right)$ plane with different photovoltage signs, depending on which of the two regions has a higher electronic density. These two regions would be separated by a single line, corresponding to the equal densities. In contrast, the thermoelectric mechanism would result in a peculiar six-fold photovoltage pattern in the $\left(V_{1}, V_{2}\right)$ plane [6]. This six-fold pattern was observed in Ref. 1.

Efficient design of optoelectronic devices requires understanding of the main mechanism of the photovoltage generation. Identification of the photothermoelectric effect as such mechanism for graphene $[1,5]$, together with the demonstration of possibility of its external control [1], paves the road for graphene-based optoelectronics. 


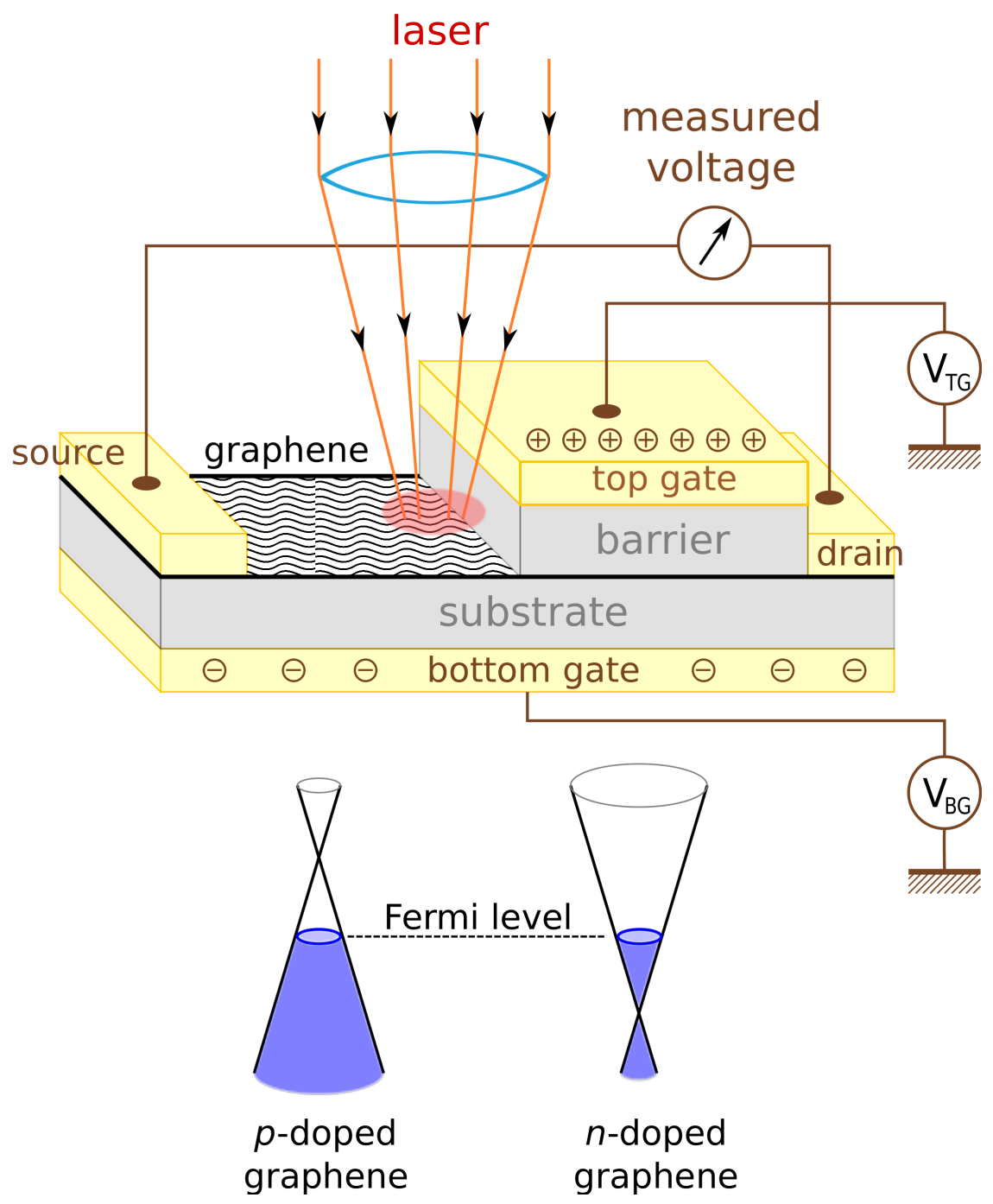

FIG. 1. Graphene-based device demonstrating the photothermoelectric effect. (Adapted from Ref. 1.) The graphene sheet is put on an insulating substrate and electrically contacted by two electrodes (source and drain). Below the substrate, the bottom gate electrode is placed under the whole graphene sheet. Above the right half of the sheet, separated by an insulating barrier, the top gate electrode is placed. The electronic density on the left is controlled by the bottom gate voltage, and the density on the right by a combination of the top and the bottom gate voltages, allowing for independent control of the densities. The junction between the two regions is illuminated by laser light, focused with a lens, and the voltage difference between the source and the drain is meausured. Bottom of the figure: schematic view of the electronic dispersion in the two regions of the graphene sheet (two Dirac cones which are vertically shifted to align the Fermi level).

[1] N. M. Gabor et al., Science 334, 648 (2011).

[2] K. S. Novoselov et al., Science 306, 666 (2004).

[3] M. Cutler and N. F. Mott, Phys. Rev. 181, 1336 (1969).

[4] Y. M. Zuev, W. Chang, and P. Kim, Phys. Rev. Lett. 102, 096807 (2009).

[5] X. Xu, N. M. Gabor, J. S. Alden, A. M. van der Zande, and P. L. McEuen, Nano Letters 10, 562-566 (2010).

[6] J. C. W. Song, M. S. Rudner, C. M. Marcus, and L. S. Levitov, Nano Lett. articles ASAP, dx.doi.org/10.1021/nl202318u. 